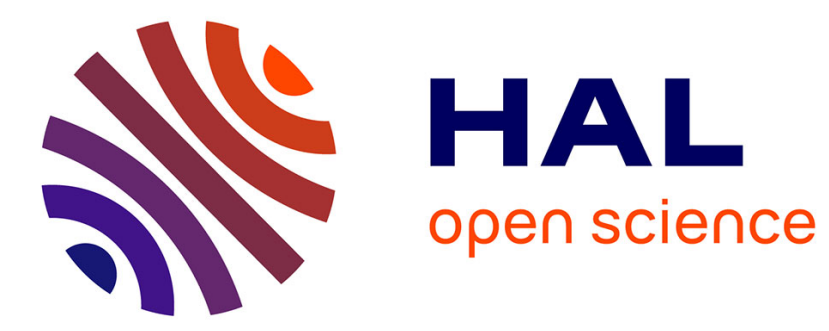

\title{
Coprimeness of Fractional Representations
}

Catherine Bonnet, Yutaka Yamamoto

\section{To cite this version:}

Catherine Bonnet, Yutaka Yamamoto. Coprimeness of Fractional Representations. 55th IEEE Conference on Decision and Control (CDC 2016), Dec 2016, Las Vegas, United States. hal-01413310

\section{HAL Id: hal-01413310 https://hal.inria.fr/hal-01413310}

Submitted on 23 Dec 2016

HAL is a multi-disciplinary open access archive for the deposit and dissemination of scientific research documents, whether they are published or not. The documents may come from teaching and research institutions in France or abroad, or from public or private research centers.
L'archive ouverte pluridisciplinaire HAL, est destinée au dépôt et à la diffusion de documents scientifiques de niveau recherche, publiés ou non, émanant des établissements d'enseignement et de recherche français ou étrangers, des laboratoires publics ou privés. 


\title{
Coprimeness of Fractional Representations
}

\author{
Catherine Bonnet ${ }^{1}$ and Yutaka Yamamoto ${ }^{2}$
}

\begin{abstract}
Coprimeness of a fractional representation plays various crucial roles in many different contexts, for example, stabilization of a given plant, minimality of a state space representation, etc. It should be noted however that coprimeness depends crucially on the choice of a ring (or algebra) where such a representation is taken, which reflects the choice of a plant, and particular problems that one studies. Such relationships are particularly delicate and interesting when dealing with infinitedimensional systems. This paper discusses various coprimeness issues for different rings, typically for $H^{\infty}$ and pseudorational transfer functions. The former is related to $H^{\infty}$-stabilizability, and the latter to controllability of behaviors. We also give some intricate examples where a seemingly non-coprime factorization indeed turns out to be a coprime factorization over $H^{\infty}$. Some future directions are also indicated.
\end{abstract}

\section{INTRODUCTION}

Coprimeness is often a central issue for various systemic studies. For example, coprimeness over the ring of stable transfer functions [14] or $H^{\infty}$ [4] gives the existence of a stabilizing controller constructed over the respective ring. Here the coprimeness of a pair $(p, q)$ means the Bézout identity

$$
p x+q y=1
$$

for some elements $x, y$ chosen in that ring.

If one changes the choice of a ring, for example, to the ring of polynomials, equation (1) translates to the minimality of a state space realization. For example, if one associates a controllable realization to the fractional representation $p / q$ of a transfer function, the Bézout condition above is equivalent to the observability of such a realization, and hence minimality (canonicity) of the realization, for the coprimeness here means that there is no redundant mode generated by the denominator $q$.

This implicitly imports the fact that nonexistence of a common factor between $p$ and $q$ implies the Bézout condition (1). It is due to the fact that the ring of polynomials is a Euclidean domain (and hence a principal ideal domain), and we see that the choice of the ring crucially determines the system properties we deal with.

The dependence on the choice of a ring becomes particularly crucial when we study infinite-dimensional systems.

\footnotetext{
${ }^{1}$ Inria, Université Paris-Saclay, L2S-CentraleSupélec, 3 rue Joliot Curie 91192 Gif-sur-Yvette cedex France Catherine.Bonneteinria.fr. This work was supported by ICODE.

${ }^{2}$ Professor Emeritus, Kyoto University, Kyoto 606-8510, Japan yyai.kyoto-u.ac.jp. This work was supported in part by the Japan Society for the Promotion of Science under Grants-in-Aid for Scientific Research No. 15H04021 and 24360163. This author wishes to thank DIGITEO, ICODE, and Laboratoire des Signaux et Systemes (L2S, UMR CNRS), CNRS-CentraleSupelec-University Paris-Sud and Inria Saclay for their financial support while part of this research was conducted.
}

There can be several non-equivalent notions of coprimeness, and also depending on the context and the choice of the ring, there are variety of results that give us deeper insights into infinite-dimensional systems.

This paper discusses such varied notions of coprimeness, and also give some examples and counterexamples, which lead us to a wider perspective for infinite-dimensional systems.

\section{PReliminaries AND PSEUdorationality}

Let $\mathscr{E}^{\prime}(\mathbb{R})$ denote the space of distributions having compact support contained in $\mathbb{R}$. $\mathscr{E}^{\prime}\left(\mathbb{R}_{-}\right)$is its subspace with support contained in the negative half line $(-\infty, 0]$. Distributions such as Dirac's delta $\delta_{a}$ placed at $a \in \mathbb{R}$, its derivative $\delta_{a}^{\prime}$ are examples of elements in $\mathscr{E}^{\prime}(\mathbb{R})$. If $a \leq 0$, then they belong to $\mathscr{E}^{\prime}\left(\mathbb{R}_{-}\right)$. An impulse response function $p \times m$ matrix $G$ (supp $G \subset[0, \infty)$ ) is said to be pseudorational ([15]) if there exist matrices $Q$ and $P$ having entries in $\mathscr{E}^{\prime}\left(\mathbb{R}_{-}\right)^{p \times p}$ and $\mathscr{E}^{\prime}\left(\mathbb{R}_{-}\right)^{p \times m}$, respectively, such that

1) $G=Q^{-1} * P$ where the inverse is taken with respect to convolution;

2 ) ord det $Q^{-1}=-\operatorname{orddet} Q$, where ord $q$ denotes the order of a distribution $q$ [11].

As an example, consider the delay-differential equation:

$$
\begin{aligned}
\dot{x}(t) & =x(t-1)+u(t) \\
y(t) & =x(t) .
\end{aligned}
$$

This can be expressed as $x=\left(\delta^{\prime}-\delta_{1}\right)^{-1} * u$, where $\delta_{a}$ is the Dirac delta distribution $\left(\delta=\delta_{0}\right)$, and $\delta^{\prime}$ its derivative. Shifting the time axis by 1 , we obtain $x=\left(\delta_{-1}^{\prime}-\delta\right)^{-1} *$ $\delta_{-1} * u$, and this is pseudorational.

In what follows, we will deal only with the SISO case, i.e., single-input and single-output case, for simplicity. For definitions and pertinent properties, see related references in [20], [15], [17], etc.

According to the Paley-Wiener theorem given in the Appendix for a pseudorational pair $(p, q)$, each component $p$ and $q$ are Laplace transformable, and have only discrete zeros. Hence for a pseudorational impulse response $G$, its Laplace transform, i.e., transfer function, $\hat{G}(s)$ is $\hat{p}(s) / \hat{q}(s)$, and hence is the ratio of entire functions satisfying the PaleyWiener estimate (15).

Definition 2.1: Let $R$ be $\mathscr{E}^{\prime}(\mathbb{R}), \mathscr{E}^{\prime}\left(\mathbb{R}_{-}\right)$or $H^{\infty}$. The pair $(p, q), p, q \in R$ is said to be spectrally coprime if $\hat{p}(s)$ and $\hat{q}(s)$ have no (finite) common zeros. It is approximately coprime if there exist sequences $\phi_{n}, \psi_{n} \in R$ such that $p *$ $\phi_{n}+q * \psi_{n} \rightarrow \delta$ in $R$. It is said to satisfy the Bézout identity 
(equation) or simply is Bézout over $R$ where $R=\mathscr{E}^{\prime}(\mathbb{R})$ or $\mathscr{E}^{\prime}\left(\mathbb{R}_{-}\right)$, if there exist $x, y \in R$ such that

$$
p * x+q * y=\delta
$$

When $R=H^{\infty}$, this translates in the Laplace domain to

$$
p x+q y=1 .
$$

\section{COPRIMENESS OVER $H^{\infty}$}

Let us start with a very simple case, which also indicates an interesting difference of coprimeness over $\mathscr{E}^{\prime}\left(\mathbb{R}_{-}\right)$and $H^{\infty}$.

Example 3.1: Consider the transfer function

$$
G(s):=\frac{1}{(s+1)\left(s\left(1-e^{-s}\right)+1\right)} .
$$

As Logemann [6] showed, this transfer function belongs to $H^{\infty}$. Since $G \in H^{\infty}$, it admits the following trivial coprime factorization

$$
p:=G, q:=1 .
$$

Note that function 1 belongs to $H^{\infty}$. The Bézout equation is trivially solved as

$$
G \cdot 0+1 \cdot 1=1
$$

Likewise, for $R=\mathscr{E}^{\prime}(\mathbb{R})$,

$$
g * 0+\delta * \delta=\delta,
$$

where $g$ denotes the inverse Laplace transform of $G$. However, over $R=\mathscr{E}^{\prime}\left(\mathbb{R}_{-}\right)$, this example exhibits a slightly different behavior. Note that the fraction (4) in itself does not give a fractional representation over $\mathscr{E}^{\prime}\left(\mathbb{R}_{-}\right)$since $e^{-s}$ or its inverse Laplace transform $\delta_{1}$ is not an element of $\mathscr{E}^{\prime}\left(\mathbb{R}_{-}\right)$, i.e., the support $\operatorname{supp} \delta_{1}=\{1\} \notin(-\infty, 0]$. We instead take an equivalent fraction

$$
G(s):=\frac{e^{s}}{(s+1)\left(s\left(e^{s}-1\right)+e^{s}\right)}
$$

or its time-domain equivalent

$$
g=\delta_{-1} *\left(\delta^{\prime}+\delta\right)^{-1} *\left[\left(\delta_{-1}^{\prime}-\delta^{\prime}\right)+\delta_{-1}\right]^{-1} .
$$

The pair $\left(\delta_{-1},\left(\delta^{\prime}+\delta\right)^{-1} *\left[\left(\delta_{-1}^{\prime}-\delta^{\prime}\right)+\delta_{-1}\right]^{-1}\right)$ is indeed Bézout, but it is not immediately obvious to exhibit a coprime factorization for this. We postpone its proof until Section IV.

\section{A. Systems with a chain of poles clustering on the imaginary} axis from the right

In this subsection we consider a particular neutral delay system with a single delay and a chain of poles asymptotic to the imaginary axis from the right with transfer function given by

$$
G(s)=\frac{1}{(s+1)\left(s+1+(s+2) e^{-s T}\right)} .
$$

We have $(s+1) /(s+2)=\alpha-\beta / s+\gamma / s+o\left(s^{-2}\right)$ with $\alpha=$ $1, \beta=-1, \gamma=2$ satisfying $\gamma / \alpha-\beta^{2} / 2>0$, so that from [9] we know that the chain of poles is in the right half-plane.
Proposition 3.2: Let $G$ be the one given by (5). Then $(N(s), D(s))$ defined by

$$
N(s)=\frac{1}{\left(-\frac{1}{3} s^{2}-\frac{2}{3} s+\frac{1}{2}\right)+\left(-\frac{1}{3} s^{2}-s-1\right) e^{-s T}}
$$

and,

$$
D(s)=\frac{(s+1)\left(s+1+(s+2) e^{-s T}\right)}{\left(-\frac{1}{3} s^{2}-\frac{2}{3} s+\frac{1}{2}\right)+\left(-\frac{1}{3} s^{2}-s-1\right) e^{-s T}}
$$

gives a Bézout factorization of $G$ over $H^{\infty}$

Proof:

Consider the quasi-polynomial $a s^{2}+b s+c+\left(d s^{2}+e s+\right.$ f) $e^{-s T}$ as a potential candidate for the denominator of $N(s)$ and $D(s)$. Write

$$
N(s)=\frac{1}{a s^{2}+b s+c+\left(d s^{2}+e s+f\right) e^{-s T}},
$$

and

$$
D(s)=\frac{(s+1)\left(s+1+(s+2) e^{-s T}\right)}{a s^{2}+b s+c+\left(d s^{2}+e s+f\right) e^{-s T}} .
$$

We then have

$$
\begin{aligned}
D(s)= & \frac{(s+1)\left(s+1+(s+2) e^{-s T}\right)}{a s^{2}+b s+c+\left(d s^{2}+e s+f\right) e^{-s T}} \\
= & \frac{1}{a}\left(1+\frac{(2 a-b) s+a-c}{d(s)}\right) \\
& +\frac{1}{a}\left(\frac{(a-d) s^{2}+s(3 a-e)+2 a-f}{d(s)}\right) e^{-s T}
\end{aligned}
$$

Taking $b=2 a, d=a$ and $e=3 a$, we see that $D(s)$ is in $H^{\infty}$ provided that $a s^{2}+b s+c+\left(d s^{2}+e s+f\right) e^{-s T}$ has no zeros in the closed right-half plane.

Using the Walton-Marshall method [7] we obtain some conditions on the coefficients to ensure that $a s^{2}+b s+c+$ $\left(d s^{2}+e s+f\right) e^{-s T}$ has no unstable poles in the closed right half-plane, for example, $a=-\frac{1}{3}, c=-\frac{1}{2}, f=-1$.

Now, it is easy to verify that $(N, D)$ satisfies the Corona condition [3] $\inf _{\operatorname{Re} s>0}(|N(s)|+|D(s)|)>0$.

Remark 3.3: The example above is somewhat counterintuitive. Note that the denominator of $G(s)$ has infinitely many zeros that approach the imaginary axis. Hence this denominator is very close to zero, at least very often, near the imaginary axis. If there is a coprime factorization $(\tilde{N}, \tilde{D})$, $\tilde{D}(s)$ should also vanish at these zeros. Furthermore, $G(s)$ has order $s^{-2}$ along the imaginary axis as $j \omega(s=j \omega)$ goes to infinity, hence it looks like $\tilde{N}(s)$ should share this order $s^{-2}$ along the imaginary axis. Since the zeros of $\tilde{D}(s)$ become closer to the imaginary axis, it may appear that $\tilde{N}(s)$ should also go to zero with order $s^{-2}$ along these zeros of $\tilde{D}(s)$. Hence it may seem that $\tilde{N}$ and $\tilde{D}$ cancel at infinity, and contradict the Corona condition. From this one may conclude that there exists no coprime factorization for this $G$. However, as we have seen above, there is indeed a coprime factorization over $H^{\infty}$. By plotting the values of $N(s)$ at these zeros, one sees that $N(s)$ is indeed bounded from below there. (Here $N$ may be close to zero along the imaginary axis, but not necessarily at the zeros of $D$.) See Figure 1 . (The zeros 
of $D(s)$, which are very close to the imaginary axis, are not shown there; the figure shows that $N(s)$ assume values quite far from zero at the zeros of $D$.)

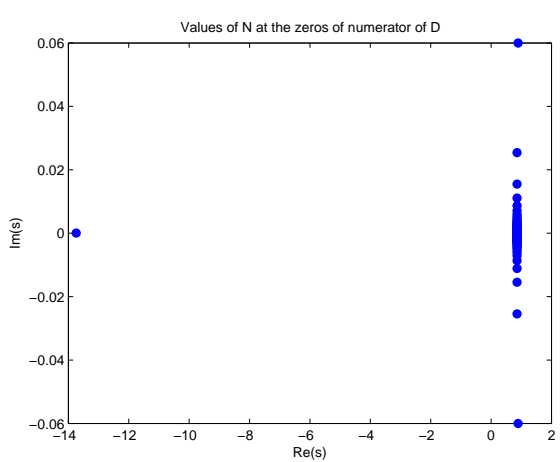

Fig. 1. Values of $N(s)$ at the zeros of $D(s)$

This example has poles clustering on the imaginary axis from the right, i.e., from the unstable side. Is there any difference if the poles approach the imaginary axis from the left? The next example considers such a case.

B. Systems with a chain of poles clustering on the imaginary axis from the left

We also give another neutral delay system with one delay and a chain of poles asymptotic to the imaginary axis from the left with transfer function given by

$$
G(s)=\frac{1}{(s+1)\left(s-1+s e^{-s T}\right)}
$$

We have $(s-1) / s=\alpha+\beta / s+\gamma / s+o\left(s^{-2}\right)$ with $\alpha=$ $1, \beta=-1, \gamma=0$ satisfying $\gamma / \alpha-\beta^{2} / 2>0$, so that from [9] we see that the chain of poles is in the left half-plane. However, $G$ may possess unstable poles of small modulus and there is also a need to determine a coprime factorization for $G$.

Proposition 3.4: Let $G$ be given by (6). The following $(N(s), D(s))$

$$
N(s)=\frac{1}{\left(s^{2}-\frac{3}{2}\right)+\left(s^{2}+s-\frac{3}{4}\right) e^{-s T}}
$$

and,

$$
D(s)=\frac{s^{2}-1+\left(s^{2}+s\right) e^{-s T}}{\left(s^{2}-\frac{3}{2}\right)+\left(s^{2}+s-\frac{3}{4}\right) e^{-s T}}
$$

gives a coprime factorization of $G$ over $H^{\infty}$.

Proof:

Consider the quasi-polynomial $d(s)=a s^{2}+b s+c+\left(d s^{2}+\right.$ $e s+f) e^{-s T}$ as a potential candidate for the denominator of $N(s)$ and $D(s)$. Write

$$
N(s)=\frac{1}{a s^{2}+b s+c+\left(d s^{2}+e s+f\right) e^{-s T}},
$$

and

$$
D(s)=\frac{(s+1)\left(s-1+s e^{-s T}\right)}{a s^{2}+b s+c+\left(d s^{2}+e s+f\right) e^{-s T}} .
$$

We then have

$$
\begin{aligned}
D(s)= & \frac{(s+1)\left(s-1+s e^{-s T}\right)}{a s^{2}+b s+c+\left(d s^{2}+e s+f\right) e^{-s T}} \\
= & \frac{1}{a}\left(1+\frac{-b s-c-a}{d(s)}\right) \\
& +\frac{1}{a}\left(\frac{(a-d) s^{2}+s(a-e)+2 a-f}{d(s)}\right) e^{-s T}
\end{aligned}
$$

The conditions $b=0, d=a, e=a$ will ensure that $D(s)$ is in $H^{\infty}$ if $d(s)$ has no unstable zeros.

The coefficients $a=1, c=-\frac{3}{2}, f=-\frac{3}{4}$ ensure that $d(s)$ has no zeros in the closed right half-plane. The rest is the same as the previous subsection.

By plotting the values of $N(s)$ at the zeros of $D(s)$ we see again that their values are quite distant from zero. See Figure 2 below.

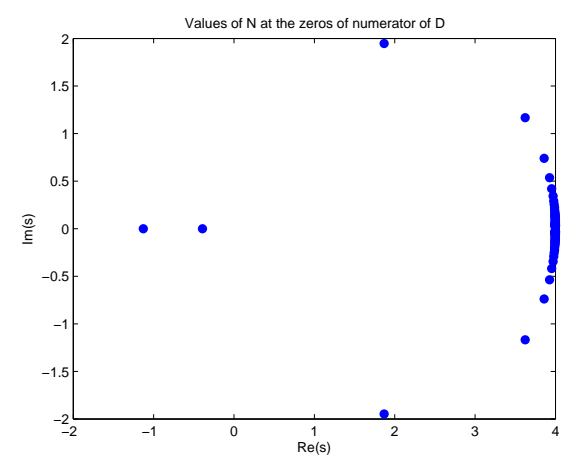

Fig. 2. Values of $N(s)$ at the zeros of $D(s)$

\section{COPRIMENESS OVER $\mathscr{E}^{\prime}(\mathbb{R})$ AND $\mathscr{E}^{\prime}\left(\mathbb{R}_{-}\right)$}

We first deal with $\mathscr{E}^{\prime}\left(\mathbb{R}_{-}\right)$. The following facts summarize the results so far obtained in [17], [19] etc.

Let $(p, q), p, q \in \mathscr{E}^{\prime}\left(\mathbb{R}_{-}\right)$be a pseudorational pair. To state the results with some rigor, we need some preliminary definitions. Let $\Omega:=\lim L^{2}[-n, 0]$ be the inductive limit of the spaces $\left\{L^{2}[-n, 0]\right\}_{n>0}$. it is the space of inputs with bounded support. Dually, $\Gamma:=L_{l o c}^{2}[0, \infty)$ is the space of all locally Lebesgue square integrable functions with obvious family of seminorms. This is the projective limit of spaces $\left\{L^{2}[0, n]\right\}_{n>0} . \Omega$ is the space of past inputs, and $\Gamma$ is the space of future outputs, with the understanding that the present time is 0 . These spaces are equipped with the following natural left shift semigroups:

$$
\begin{aligned}
& \left(\sigma_{t} \omega\right)(s):= \begin{cases}\omega(s+t), & s \leq-t, \\
0, & -t<s \leq 0,\end{cases} \\
& \omega \in \Omega, t \geq 0, s \leq 0 \text {. } \\
& \left(\sigma_{t} \gamma\right)(s):=\gamma(s+t), \quad \gamma \in \Gamma, t \geq 0, s \geq 0 .
\end{aligned}
$$

The input/output map associated with $(p, q)$ is defined as

$$
f(\omega): \Omega \rightarrow \Gamma: \omega \mapsto \pi\left(q^{-1} * p * \omega\right),
$$

where $\pi \psi:=\left.\psi\right|_{[0, \infty)}$ is the truncation at 0 . 
Now define

$$
X^{q}:=\{x \in \Gamma \mid \operatorname{supp}(q * x) \subset(-\infty, 0]\} .
$$

$X^{q}$ is a left-shift invariant subspace of $\Gamma$, and we can take this left-shift operator as the state transition semigroup. The " $A$ " operator is the infinitesimal generator of this semigroup, and the remaining $B$ and $C$ are defined suitably; see [15] for detailed definitions. We denote this system by $\Sigma^{q, p}$.

The following facts hold:

Facts 4.1: 1) Every eigenmode of $\Sigma^{q, p}$ is controllable if and only if $(p, q)$ is spectrally coprime.

2) The pair is approximately coprime if and only if

- $(p, q)$ is spectrally coprime, and

- $\max \{r(q), r(p)\}=0$

where $r(q)$ denotes $\sup \{t \in \operatorname{supp} q\}$.

This result immediately leads to the observation that there indeed exists a pair $(p, q)$ that is spectrally coprime but not approximately coprime. Indeed, see the following example.

Example 4.2: Let $\hat{p}:=1, \hat{q}:=e^{s}-1$. Clearly

$$
1 \cdot 1+\left(e^{s}-1\right) \cdot 0=1 \text {. }
$$

On the other hand, if we multiply $e^{s}$ to both $\hat{p}$ and $\hat{q}$, they have a common factor $e^{s}$. Since $e^{s}$ is not invertible in $\mathscr{E}^{\prime}\left(\mathbb{R}_{-}\right)$, the pair $\left(e^{s} \hat{p}, e^{s} \hat{q}\right)$ cannot be approximately coprime.

It is interesting to note that condition

$$
r(q)=0
$$

means eigenfunction completeness of $\Sigma^{q, p}$. If this holds, spectral coprimeness obviously implies approximate controllability. In fact, the condition

$$
\max \{r(q), r(p)\}=0
$$

means that system $\Sigma^{q, p}$ can be made eigenfunction complete by a unity feedback in itself [17]. Since feedback does not change controllability, this immediately implies that spectral controllability yields approximate controllability.

\section{A. Bézout identity over $\mathscr{E}^{\prime}\left(\mathbb{R}_{-}\right)$}

The strongest notion of coprimeness is the Bézout condition. Clearly

Bézout $\Rightarrow$ approximately coprime $\Rightarrow$ spectrally coprime

and each implication is strict, i.e., the converse does not hold. As we have seen already, giving an example that approximate comprimeness does not imply a Bézout identity is not trivial. We here give some examples.

Example 4.3: Let $(p, q)$ admit the following Bézout identity.

$$
p * x+q * y=\delta
$$

This yields

$$
q^{-1}=q^{-1} * p * x+y
$$

Then we have

$$
q^{-1}=\pi\left(q^{-1} * p * x\right)
$$

because $\pi y=0$. In other words, with an extended input $x \in$ $\mathscr{E}^{\prime}\left(\mathbb{R}_{-}\right), q^{-1}$ is reachable from 0 .
Now if we take $p$ to be a $C^{\infty}$ function with support in $[-T, 0]$, and let $q:=\delta_{-T}$. It follows that

$$
\delta_{T}=\pi\left(\delta_{T} * p * x\right),
$$

but for any $x \in \mathscr{E}^{\prime}\left(\mathbb{R}_{-}\right)$, the right-hand side is always in $C^{\infty}$, hence it cannot be $\delta_{T}$. Hence this pair does not admit a Bézout identity.

Example 4.4: Consider $p:=e^{s}, q:=e^{s}+1$. The pair is Bézout. Indeed,

$$
e^{s} \cdot(1)+\left(e^{s}-1\right) \cdot(-1)=1 .
$$

We also note that a "Corona-like" condition

$$
\inf _{s \in \mathbb{C}}\left\{\left|e^{s}\right|+\left|e^{s}-1\right|\right\}=1
$$

holds.

If we multiply $s$ to $e^{s}$ in the second factor, we have a more delicate example.

Example 4.5: Consider $p:=e^{s}, q:=s e^{s}-1$. Then as

$$
e^{s} \cdot(s)+\left(s e^{s}-1\right) \cdot(-1)=1
$$

shows, this pair is Bézout. Now note that $s e^{s}-1$ has infinitely many zeros that goes to infinity. Along these zeros $\lambda_{n}, n=$ $1,2, \ldots, e^{\lambda_{n}}$ goes to zero with order $1 / \lambda_{n}$. Hence at these zeros $\lambda_{n}$,

$$
\inf _{s=\lambda_{n}}\left\{\left|e^{s}\right|+\left|s e^{s}-1\right|\right\}=0,
$$

and a "Corona-like" condition fails.

Unlike the previous example, $q$ has the first-order differentiation $s$, and this prohibits a routine application of a "Corona-like" condition. This is due to the fact that distributions are locally of finite order, and it can allow multiplication by polynomials (and other distributions of finite order). In fact,

$$
\left|s \cdot e^{s}\right|=1>0, \quad s=\lambda_{n},
$$

and this multiplication of $s$ "saves" the asymptotic cancellation at infinity. The following theorem indeed assures this.

We quote the following theorem from [19]:

Theorem 4.6: Let $(p, q)$ be pseudorational with $p, q \in$ $\mathscr{E}^{\prime}\left(\mathbb{R}_{-}\right)$such that $r(q)=0$. Suppose that the algebraic multiplicities of $\lambda$ in $\Lambda:=\{\lambda \in \mathbb{C} \mid \hat{q}(\lambda)=0\}$, i.e., the zeros of $\hat{q}$ are globally bounded, say by $m$. If

$$
\left|(1+|\lambda|)^{m} \hat{p}(\lambda)\right| \geq c, \quad \forall \lambda \in \Lambda,
$$

then the pair $(p, q)$ is Bézout.

In Example 4.5, the order of $q$ is 1 , and the multiplicities of the zeros of $\hat{q}(s)$ is bounded by 1 . We have just seen (11) is satisfied.

Example 4.7: Let us return to Example 3.1. Here $\hat{p}(s)=e^{s}$ and $\hat{q}(s)=s\left(e^{s}-1\right)+e^{s}$. As with other examples of neutral systems, $\hat{q}(s)$ has a chain of zeros approaching the imaginary axis. Denote these zeros of $\hat{q}(s)$ by $\lambda_{n}, n=1,2, \ldots$, and because of this property, $\inf \left\{\operatorname{Re} \lambda_{n}\right\} \geq a$ for some $a \in \mathbb{R}$. On the other hand, $\left|e^{s}\right| \geq e^{\operatorname{Re} a}>0$, so that by Theorem 4.6 $(p, q)$ is a Bézout pair. 


\section{B. Coprimeness over $\mathscr{E}^{\prime}(\mathbb{R})$}

We now consider the case of $\mathscr{E}^{\prime}(\mathbb{R})$. While $\mathscr{E}^{\prime}\left(\mathbb{R}_{-}\right)$is closely related to the realization $\Sigma^{q, p}$, this case $\mathscr{E}^{\prime}(\mathbb{R})$ is closer to behavioral system theory. Also, while $\delta_{a}$ is not unimodular in $\mathscr{E}^{\prime}\left(\mathbb{R}_{-}\right)$unless $a=0$, it is indeed unimodular in $\mathscr{E}^{\prime}(\mathbb{R})$ because its inverse is $\delta_{-a}$. This has some important consequences for coprimeness notions. For example, spectral coprimeness is equivalent to approximate coprimeness over $\mathscr{E}^{\prime}(\mathbb{R})$. Indeed, recall that the difference between the two notions is related to eigenfunction completeness, and this is further related to $r(p)$ or $r(q)$. The notion of approximate coprimeness is invariant by shifts in $\mathscr{E}^{\prime}(\mathbb{R})$, one can conclude approximate coprimeness from spectral coprimeness.

We start with the following definition:

Definition 4.8: Let $(p, q)$ be pseudorational with $p, q \in$ $\mathscr{E}^{\prime}(\mathbb{R})$. The distributional behavior $\mathscr{B}_{\mathscr{D}^{\prime}}$ defined by $(p, q)$ is given by

$$
\mathscr{B}_{\mathscr{D}^{\prime}}:=\left\{w \in\left(\mathscr{D}^{\prime}\right)^{2} \mid[p q] * w=0\right\} .
$$

For controllability, we employ the following definition:

Definition 4.9: Let $\mathscr{B}_{\mathscr{D}^{\prime}}$ be the distributional behavior (12). $\mathscr{B}_{\mathscr{D}^{\prime}}$ is said to be distributionally controllable if for every pair $w_{1}, w_{2} \in \mathscr{B}_{\mathscr{D}^{\prime}}$, there exists $T \geq 0$ and $w \in \mathscr{B}_{\mathscr{D}^{\prime}}$, such that $\left.w\right|_{(-\infty, 0)}=w_{1}$ on $(-\infty, 0)$, and $\left.w\right|_{(T, \infty)}=\sigma_{-T} w_{2}$ on $(T, \infty)$.

Then we have the following [21]:

Theorem 4.10: Let $(p, q), p, q \in \mathscr{E}^{\prime}(\mathbb{R})$ be pseudorational, and let $\mathscr{B}_{\mathscr{D}^{\prime}}$ be as defined above in (12). Then $\mathscr{B}_{\mathscr{D}^{\prime}}$ is distributionally controllable if and only if $(p, q)$ is a Bézout pair, i.e., there exist $x, y \in \mathscr{E}^{\prime}(\mathbb{R})$ such that $p * x+q * y=\delta$.

This is a special case of a more general theorem given in [21]. For the proof, see [21].

Now generalizing Theorem 4.6, we have the following:

Proposition 4.11: Let $p, q$ be as above, and suppose that

$$
r(p)=a, r(q)=b .
$$

Suppose that the multiplicities of zeros $\lambda \in \Lambda$ are globally bounded and

$$
\left|(1+|\lambda|)^{m}\left(\delta_{-a} * p\right)^{\gamma}(\lambda)\right|=\left|(1+|\lambda|)^{m} e^{a \lambda} \hat{p}(\lambda)\right| \geq c, \quad \forall \lambda \in \Lambda
$$

for some $m$ and $c>0$. Then $(p, q)$ is a Bézout pair in $\mathscr{E}^{\prime}(\mathbb{R})$. Proof Condition (13) implies $\delta_{-a} * p, \delta_{-b} * q \in \mathscr{E}^{\prime}\left(\mathbb{R}_{-}\right)$, and $r\left(\delta_{-b} * q\right)=0$. Since

$$
\left|(1+|\lambda|)^{m}\left(\delta_{-a} * p\right)^{\gamma}(\lambda)\right| \geq c \quad \forall \lambda \in \Lambda
$$

by (14), we can apply Theorem 4.6 to $\delta_{-a} * p, \delta_{-b} * q$ to obtain

$$
\delta_{-a} * p * x+\delta_{-b} * q * y=\delta
$$

for some $x, y \in \mathscr{E}^{\prime}\left(\mathbb{R}_{-}\right)$. This yields

$$
p *\left(\delta_{-a} * x\right)+q *\left(\delta_{-b} * y\right)=\delta
$$

as desired.

Example 4.12: Consider the pair $p(s)=e^{s}$ and $q(s):=$ $s e^{2 s}-e^{s}$. Note that there is no common zero between $p(s)$ and $q(s)$, and hence the pair is spectrally coprime. On the other hand, it is not even approximately coprime over
$\mathscr{E}^{\prime}\left(\mathbb{R}_{-}\right)$since it has a common factor $e^{s}$, and hence not Bézout over $\mathscr{E}^{\prime}\left(\mathbb{R}_{-}\right)$. However, it is coprime over $\mathscr{E}^{\prime}(\mathbb{R})$ as

$$
e^{s} \cdot s e^{-s}+\left(s e^{2 s}-e^{s}\right) \cdot\left(-e^{-s}\right)=1
$$

Clearly this is possible because $\delta_{-1}$ (with Laplace transform $e^{s}$ ) is unimodular in $\mathscr{E}^{\prime}(\mathbb{R})$ (but not in $\mathscr{E}^{\prime}\left(\mathbb{R}_{-}\right)$) with inverse $\delta_{1}$.

Remark 4.13: It is worth noting that [5], [10] have proven that systems with commensurable delays are controllable if and only if $(\hat{p}(s), \hat{q}(s))$ has no cancellation in $\mathbb{C}$. In light of Proposition 4.11, this may appear strange because the proposition generally requires "no cancellation at infinity." However, the very nature of delay systems with commensurable delays makes it possible to conclude that any cancellation at infinity can be suitably "removed" by applying multiplication by a polynomial in $s$, which is exactly the case in Example 4.5. See [21] for a more detailed discussion.

\section{APPENDIX}

The following Paley-Wiener theorem is fundamental for the Laplace transform of elements in $\mathscr{E}^{\prime}(\mathbb{R})$.

Theorem 1.1 ([11]): A complex analytic function $f(s)$ is the Laplace transform of a distribution $\phi \in \mathscr{E}^{\prime}(\mathbb{R})$ if and only if $f(s)$ is an entire function that satisfies the following growth estimate for some $C>0, a>0$ and integer $m \geq 0$ :

$$
|f(s)| \leq C(1+|s|)^{m} e^{a|\operatorname{Re} s|} .
$$

In particular, $f(s)=\hat{\phi}(s)$ for some $\phi \in \mathscr{E}^{\prime}\left(\mathbb{R}_{-}\right)$if and only if it satisfies the estimate

$$
\begin{aligned}
|\hat{f}(s)| & \leq C(1+|s|)^{m} e^{a \operatorname{Re} s}, \operatorname{Re} s \geq 0 \\
& \leq C(1+|s|)^{m}, \operatorname{Re} s \leq 0
\end{aligned}
$$

for some $C>0, a>0$ and integer $m \geq 0$. In this case, the support of $\phi$ is contained in $[-a, 0]$

We will refer to (15) as the Paley-Wiener estimate, and the class of functions satisfying this estimate as the Paley-Wiener class.

The zeros of $\hat{f}(s)$ in the Paley-Wiener class are discrete, and each zero has a finite multiplicity. This in particular implies the following Hadamard factorization for $\hat{f}(s)$ [1] in terms of the zeros of $f$ :

$$
\hat{f}(s)=c s^{k} e^{a s} \prod_{n=1}^{\infty}\left(1-\frac{s}{\lambda_{n}}\right) \exp \left(\frac{s}{\lambda_{n}}\right) .
$$

Since there are no finite accumulation point for $\left\{\lambda_{n}\right\}, \lambda_{n} \rightarrow \infty$ as $n \rightarrow \infty$.

\section{REFERENCES}

[1] R. P. Boas Jr., Entire Functions, Academic Press, 1954.

[2] C. Bonnet, A.R. Fioravanti and J. R. Partington, "Stability of neutral systems with commensurate delays and poles asymptotic to the imaginary axis," SIAM J. Control Optimiz., vol. 49, No. 2, pp. 498-516.

[3] J. B. Garnett, Bounded Analytic Functions, Academic Press, 1981.

[4] T. T. Georgiou and M. Smith, "Graphs, causality, and stabilizability: Linear, shift-invariant systems on $\mathscr{L}_{2}[0, \infty)$," Math. Control, Signals and Systems, vol. 3, pp. 195-223.

[5] H. Glüsing-Lüerssen, "A behavioral approach to delay-differential systems," SIAM J. Control \& Optimiz., 35, No. 2: 480-499, 1997. 
[6] H. Logemann, "Transfer-function conditions for the stability of neutral and Volterra integrodifferential systems," IMA J. Math. Control \& Information, 3: 9-19, 1986.

[7] J. E. Marshall and H. Górecki and K. Walton and A. Korytowski, Time Delay Systems: Stability and Performance Criteria with Applications, Ellis Horwood, 1992.

[8] L. H. V. Nguyen and C. Bonnet, "Stabilization of some fractional neutral delay systems which possibly possess an infinite number of unstable poles," ADDS series, Springer, 2014.

[9] J. R. Partington and C. Bonnet, " $H_{\infty}$ and BIBO stabilization of delay systems of neutral type," Systems and Control Letters, vol. 52, pp. 283288, 2004.

[10] P. Rocha and J. C. Willems, "Behavioral controllability of delaydifferential systems," SIAM J. Control \& Optimiz., 35: 254-264, 1997.

[11] L. Schwartz, Théorie des Distribution, Hermann, 1966.

[12] M.C. Smith, "On stabilization and the existence of coprime factorizations," IEEE Trans. Autom. Control, vol. 34, N. 9, pp. 1005-1007, 1989.

[13] F. Treves, Topological Vector Spaces, Distributions and Kernels, Academic Press, 1967.

[14] M. Vidyasagar, Control System Synthesis: A Factorization Approach, MIT Press, Cambridge, MA, 1985.

[15] Y. Yamamoto, "Pseudo-rational input/output maps and their realizations: a fractional representation approach to infinite-dimensional systems," SIAM J. Control \& Optimiz., 26: 1415-1430, 1988.

[16] Y. Yamamoto and S. Hara, "Relationships between internal and external stability with applications to a servo problem," IEEE Trans. Autom. Control, AC-33: 1044-1052, 1988.

[17] Y. Yamamoto, "Reachability of a class of infinite-dimensional linear systems: an external approach with applications to general neutral systems," SIAM J. Control \& Optimiz., 27: 217-234, 1989.

[18] Y. Yamamoto, "Equivalence of internal and external stability for a class of distributed systems," Math. Control, Signals and Systems, 4: 391-409, 1991.

[19] Y. Yamamoto, "Coprimeness in the ring of pseudorational transfer functions," Proc. 15th Mediterranean Conference on Control and Automation, 2007.

[20] Y. Yamamoto, "Pseudo-rational transfer functions-A survey of a class of infinite-dimensional systems, Proc. 46th IEEE CDC, pp. 848-853, 2007.

[21] Y. Yamamoto, "Behavioral controllability and coprimeness for pseudorational transfer functions," Syst. Control Lett., vol. 95, pp. 20-26, 2016. 